\title{
REFERENCES
}

1. R. P. Kanwal, Shock and wave surfaces for three-dimensional gas flows, Ph.D. Thesis, Indiana University, 1957.

2. - On curved shock waves in three-dimensional gas flows, Presented at the American Mathematical Society meeting in April, 1957, at Chicago, to appear in the Quarterly of Applied Mathematics.

3. - Propagation of curved shocks in pseudo-stationary three-dimensional gas flows, to appear in Illinois Journal of Mathematics.

4. A. H. Taub, Determination of flows behind stationary and pseudostationary shocks, Ann. of Math. vol. 62 (1955) pp. 300-325.

5. L. P. Eisenhart, Introduction to differential geometry, Princeton University Press, 1941, Chapters III and IV.

6. A. H. Fletcher, A. H. Taub and W. Bleakney, The Mach reflection of shock waves at nearly glancing incidence, Reviews of Modern Physics vol. 23 (1951) pp. 271-286.

Mathematics Research Center, University of Wisconsin

\section{NOTE ON A PAPER OF CIVIN AND YOOD}

\section{LEONARD E. BAUM}

It is possible to obtain stronger results than those of [1] in a small amount of space through the use of a different method. We follow the notation of [1] throughout.

Theorem 1. Let B be a complex commutative Banach algebra with countable space TI of maximal regular ideals. Let $A$ be a separating subalgebra. Then (i) if $A$ is not contained in any maximal regular ideal it is determining. Otherwise (ii) there exists a (unique) maximal regular ideal $M$ of $B$ with $\hat{A}$ dense in $\hat{M}$.

Proof. If $x \in A$ then $\mathrm{Cl}[\hat{x}(\mathfrak{T})]=\hat{x}(\mathfrak{T}) \cup\{0\}$ is a countable compact set. Then by a theorem of Lavrent'ev [2], polynomials in $z$ are uniformly dense on $\mathrm{Cl}[\hat{x}(\mathfrak{T})]$ in all continuous complex valued functions on $\mathrm{Cl}[\hat{x}(\mathfrak{T})]$ and in particular uniformly approximate $\bar{z}$ on $\mathrm{Cl}[\hat{x}(\mathfrak{T})]$, i.e. polynomials in $\hat{x}$ uniformly approximate $\hat{x}^{-}$on $\mathfrak{T}$. Then, $\mathrm{Cl}[\hat{A}]$ being a uniformly closed, separating, self adjoint algebra of continuous, complex valued functions vanishing at $\infty$ on a locally compact space $\mathscr{T}$, equals $\mathfrak{C}(\mathfrak{T})$ in case (i) or at worst some unique $\mathrm{Cl}[\widehat{\mathfrak{N}}]$ in case (ii) by the Stone-Weierstrass approximation theorem [3].

Theorem 3.3 and extensions of Theorem 3.5 and Corollary 3.6 of [1] eliminating the hypothesis of regularity and the necessity of limiting the discussion to maximal subalgebras follow as simple corollaries, e.g.

Received by the editors January 29, 1957 and, in revised form, June 15, 1957. 
COROLlary 2. The only closed separating subalgebras of a complex commutative $B^{*}$-algebra with a countable space of maximal regular ideals are these maximal regular ideals themselves.

The method of proof of Theorem 1 and the Lavrent'ev Theorem will also yield the following more general version:

THeOREM 3. If $A$ is a separating subalgebra of a complex commutative Banach algebra $B$ such that all $x \in A$ have spectrum plus $\{0\}$ nowhere dense, nonseparating sets of complex numbers, then $A$ is determining if it is not contained in a maximal regular ideal, and otherwise $\hat{A}$ is dense in $\hat{M}$ for some unique maximal regular ideal $M$ of $B$.

A number of related results are readily derivable from this theorem.

\section{REFERENCES}

1. P. Civin and B. Yood, Regular Banach algebras with a countable space of maximal regular ideals, Proc. Amer. Math. Soc. vol. 7 (1956) pp. 1005-1010.

2. M. A. Lavrent'ev, Sur les fonctions d'une variable complexe représentables par des séries de polynômes, Paris, Hermann, 1936.

3. M. H. Stone, The generalized Weierstrass approximation theorem, Math. Mag. vol. 21 (1948) pp. 167-184, 237-254.

HARVARD UNIVERSITY 